Supporting information for

\title{
Influence of Crystal Water on Crystal Structure, Electronic Structure, Band Structure and Charge Separation of $\mathrm{WO}_{3} \cdot 2 \mathrm{H}_{2} \mathrm{O}$ Nanosheets
}

Shaoqian Shi ${ }^{1}$, Fei Teng ${ }^{* 1}$, Weiyi Hao ${ }^{1}$, Wenhao $\mathrm{Gu}^{1}$, Zhicheng Yang ${ }^{1}$, Fangdi Zhao ${ }^{2}$

${ }^{1}$ Jiangsu Engineering and Technology Research Centre of Environmental Cleaning Materials (ECM), Collaborative Innovation Centre of Atmospheric Environment and Equipment Technology (CICAEET), Jiangsu Key Laboratory of Atmospheric Environment Monitoring and Pollution Control (AEMPC), Nanjing University of Information Science and Technology, 219 Ningliu Road, Nanjing 210044, China. E-mail:tfwd@163.com (F.Teng).

${ }^{2}$ Nanjing Fangzheng Construction Quality Testing Co., Ltd, Nanjing 210003, China 


\section{Experimental}

\section{Sample Preparation}

All reagents were of analytical grade, purchased separately from Shanghai Chemical Reagents Company of China, and were used without further purification.

$\mathrm{WO}_{3} \cdot 2 \mathrm{H}_{2} \mathrm{O}$ is synthesized by a simple precipitation method. Typically, $400 \mathrm{mg}$ of $\mathrm{Na}_{2} \mathrm{WO}_{4} \cdot 2 \mathrm{H}_{2} \mathrm{O}$ were added to $300 \mathrm{~mL}$ of $4.8 \mathrm{M}$ nitric acid solution at room temperature. After the mixture was stirred for $72 \mathrm{~h}$, the light yellow precipitates were separated by centrifuging and washed with deionized water for several times. Finally, the powders were dried overnight at room temperature.

The $\mathrm{WO}_{3}$ sample was obtained by calcining $\mathrm{WO}_{3} \cdot 2 \mathrm{H}_{2} \mathrm{O}$ powders at $300{ }^{\circ} \mathrm{C}$ for $2 \mathrm{~h}$.

\section{Characterization}

The crystal structures of the samples were determined by X-ray powder polycrystalline diffractometer (Rigaku D/max-2550VB), using graphite monochromatized $\mathrm{Cu} \mathrm{K}_{\alpha}$ radiation $(\lambda=$ $0.154 \mathrm{~nm}$ ), operating at $40 \mathrm{kV}$ and $50 \mathrm{~mA}$. The XRD patterns were obtained in the range of 20-80 (20) at a scanning rate of $7^{\circ} \mathrm{min}^{-1}$. Nitrogen sorption isotherms were performed at $77 \mathrm{~K}$ and $<10^{-4}$ bar on a Micromeritics ASAP2010 gas adsorption analyzer. Each sample was degassed at $150^{\circ} \mathrm{C}$ for $5 \mathrm{~h}$ before measurements. Surface area was calculated by the Brunauer-Emmett-Teller (BET) method. Fourier transform infrared spectra (FT-IR) were recorded on a Fourier transform infrared spectra (FT-IR, KBr disk method; Termo Scientifc Nicolet iS5) at the wavenumber range of 400$4000 \mathrm{~cm}^{-1}$. The samples were characterized on a scanning electron microscope (SEM, Hitachi SU-1510) with an acceleration voltage of $15 \mathrm{keV}$. The samples were coated with 5-nm-thick gold layer before observations. The fine surface structures of the samples were determined by high-resolution transmission electron microscopy (HRTEM, JEOL JEM-2100F) equipped with an electron diffraction (ED) attachment with an acceleration voltage of $200 \mathrm{kV}$. X-ray photoelectron spectroscopy (XPS) measurements were done on a VG ESCALAB MKII XPS system with Mg $\mathrm{K}_{\alpha}$ source and a charge neutralizer. All the binding energies were referenced to the $\mathrm{C} 1 \mathrm{~s}$ peak at 284.8 $\mathrm{eV}$ of surface adventitious carbon. UV-vis diffused reflectance spectra (UV-DRS) of the samples were obtained using a UV-vis spectrophotometer (UV-2550, Shimadzu, Japan). Each sample was degassed at $120{ }^{\circ} \mathrm{C}$ for $5 \mathrm{~h}$ before measurements. $\mathrm{BaSO}_{4}$ was used as a reflectance standard in a UV-vis diffuse reflectance experiment. Photoluminescence (PL) spectra were measured on a fluorescence spectrophotometer (Japan, Shimadzu RF-5301PC) with the $340 \mathrm{~nm}$ excitation line of a Xe lamp as the excitation source.

\section{Electrochemistry measurement}

An electrochemical system (CHI-660B, China) was employed to measure the photocurrents and electrochemical impedance spectroscopy (EIS). Electrochemical impedance spectroscopy (EIS) was performed from $0.1 \mathrm{~Hz}$ to $100 \mathrm{kHz}$, at an open circuit potential of $0.1 \mathrm{~V}$ and an 
alternating current (AC) voltage amplitude of $5 \mathrm{mV}$. The data were analyzed by ZSimWin software. Photocurrent measurements were carried out under visible light $(\lambda \geq 420 \mathrm{~nm})$ in a conventional three-electrode system, in which indium-tin oxide (ITO) glass was used as the current collector to fabricate photo electrode, and $0.5 \mathrm{M} \mathrm{Na}_{2} \mathrm{SO}_{4}$ was used as the electrolyte solution. ITO photo electrodes were prepared by a dip-coating method, which was implemented as the photo anode in a photoelectrochemical cell (PEC). The Mott-Schottky method was applied to measure the flat potential $\left(\mathrm{E}_{\mathrm{fb}}\right)$ of semiconductor, which are immersed in $0.5 \mathrm{~mol} \mathrm{~L}-1 \mathrm{Na}_{2} \mathrm{SO}_{4}$ solution $(\mathrm{pH}=7)$, which is carried out in conventional three electrode cells using a CHI660D electrochemical workstation (Shanghai Chenhua Instrument Co., Ltd., Shanghai, China).

The surface photovoltage (SPV) measurement system is consisted of a monochromatic light source, a lock-in amplifier (SR830-DSP) with a light chopper (SR540), a photovoltaic cell and a computer. The monochromatic light is provided by a $500 \mathrm{~W}$ xenon lamp (CHFXQ500 W, Global Xenon Lamp Power) and a double-prism monochromator (Zolix SBP500). During the SPV measurement, the samples were test without further treatment. While the measurement of surface photovoltage was performed, the contact between the samples and the indium tin oxide (ITO) electrode was not ohmic. The construction of the photovoltaic cell is a sandwich like structure, which consist of steel timber, sample and ITO. We placed the powders on the steel timber electrode, then, the powder was pressed to obtain a film with ITO electrode.

\section{Evaluation of photocatalytic activity}

The photocatalytic activity of the sample was evaluated by the degradation of Methylene blue (MB) aqueous solution under visible light $(\lambda \geq 420 \mathrm{~nm}$ ), using a $300 \mathrm{~W}$ Xe arc lamp (CEL-HXF $300)$. The reaction system was placed in a sealed black box with the top opened, and the distance from reaction system to light source was maintained at $15 \mathrm{~cm}$. The total irradiation power impinging on sample was determined to be $0.170 \mathrm{Wcm}^{-2}$. The powders $(100 \mathrm{mg})$ were dispersed in $200 \mathrm{~mL}$ of $4.5 \mathrm{mg} / \mathrm{L} \mathrm{MB}$ aqueous solution in a Pyrex beaker at room temperature. Before lamp was turned on, the suspension was continuously stirred for $30 \mathrm{~min}$ in the dark to ensure the establishment of an adsorption-desorption equilibrium between the powders and MB. At a time interval, $3 \mathrm{~mL}$ of solution was collected by pipette, and subsequently centrifuged to remove the powders. UV-vis absorption spectra were recorded on a Spectrumlab 722sp spectrophotometer to determine the concentration of MB. The degradation reaction can be expressed by an apparent first-order reaction kinetic, which could be calculated using the following equation:

$$
\ln \left(\mathrm{C}_{0} / \mathrm{C}\right)=k_{a} \times \mathrm{t}
$$

where $\mathrm{C}_{0}$ is the concentration of the initial MB solution, $\mathrm{C}$ is the concentration of MB at $t$-min irradiation and $k_{a}$ represents the apparent first-order reaction kinetic constant. 


\section{Figure S1}

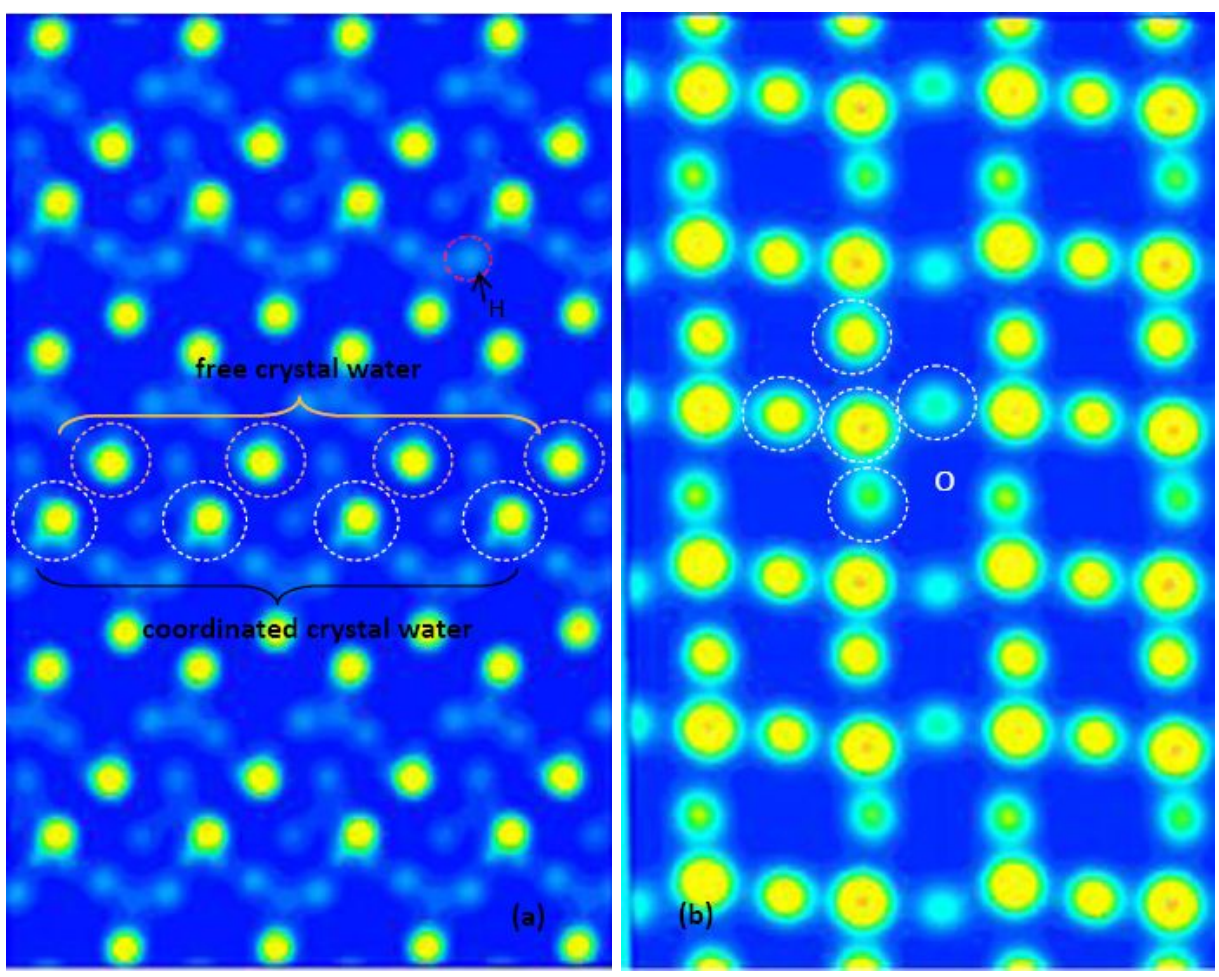

Figure $\mathrm{S} 1$ electron density contour plots of oxygen atoms: (a) $\mathrm{WO}_{3} \cdot 2 \mathrm{H}_{2} \mathrm{O}$; (b) $\mathrm{WO}_{3}$ 


\section{Figure S2}
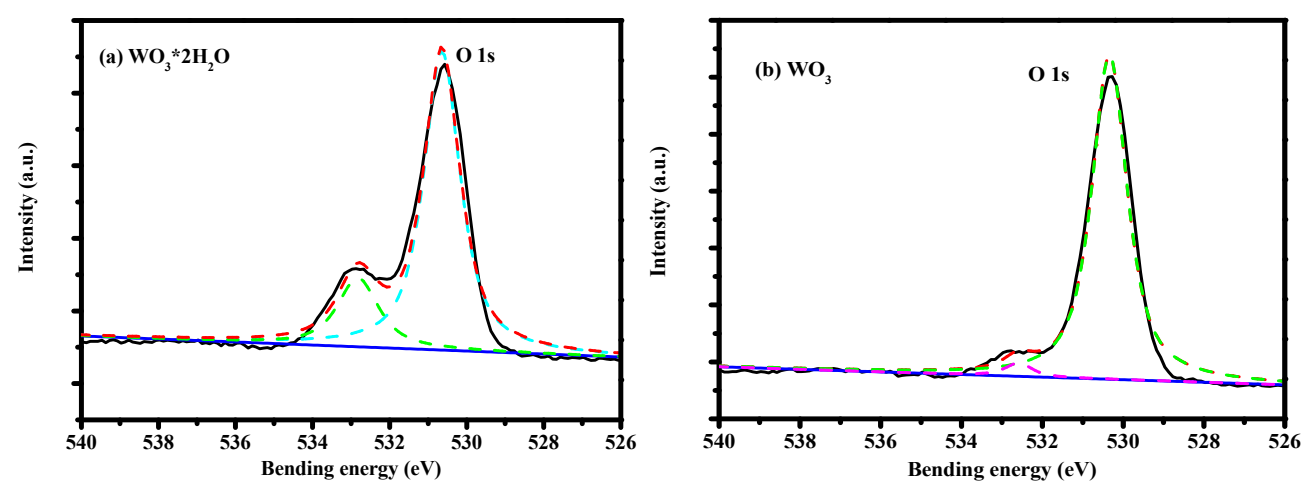

Figure S2 The fitted X-ray photoelectron spectra (XPS) of O 1s 
Figure S3

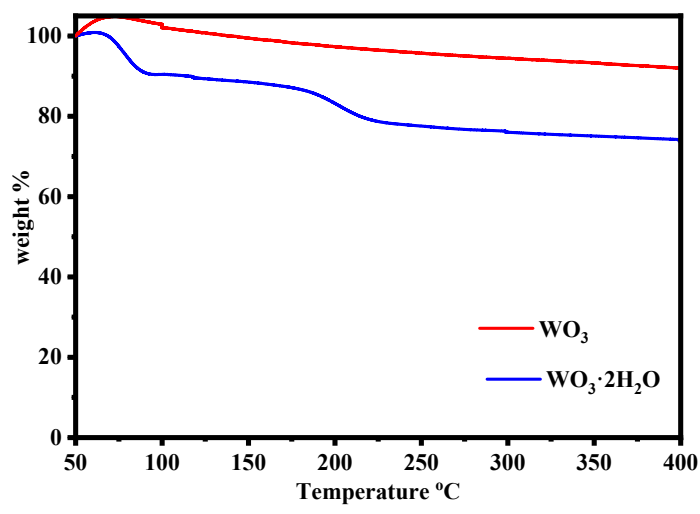

Figure $\mathbf{S 3}$ (a) Thermogravimetry plots of $\mathrm{WO}_{3} \cdot 2 \mathrm{H}_{2} \mathrm{O}$ and $\mathrm{WO}_{3}$ samples. 


\section{Figure S4}

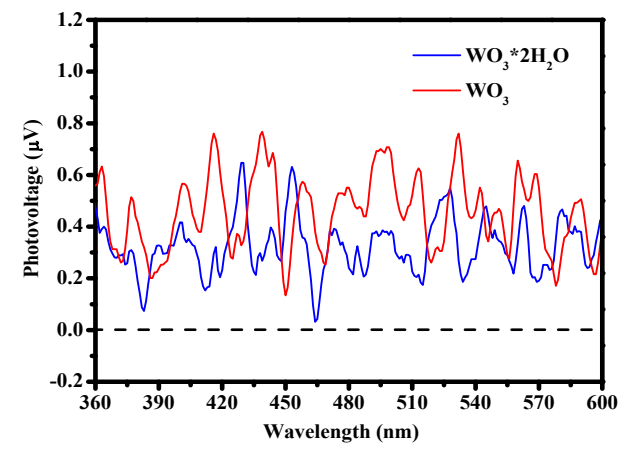

Figure S4 surface photovoltage (SPV) spectra 
Figue S5
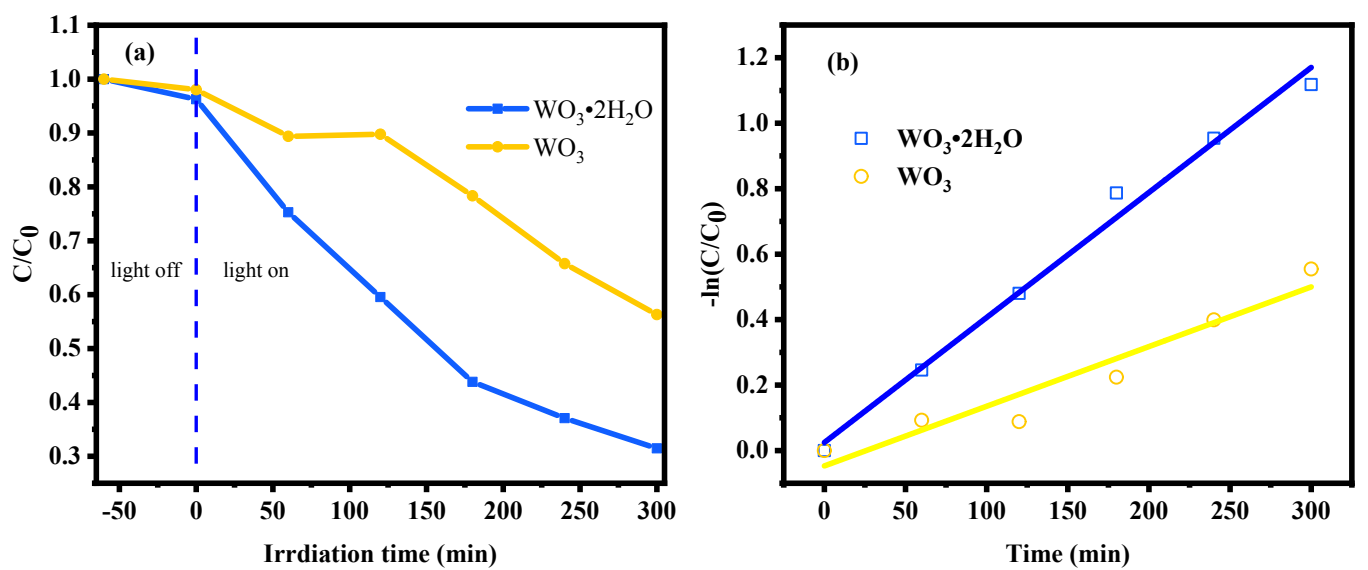

Figure S5 Degradation curves (a) And reaction kinetic curves (b) of 2-nitrophenol over the samples under visible light irradiation $(\lambda \geq 420 \mathrm{~nm})$. 


\section{Figure S6}
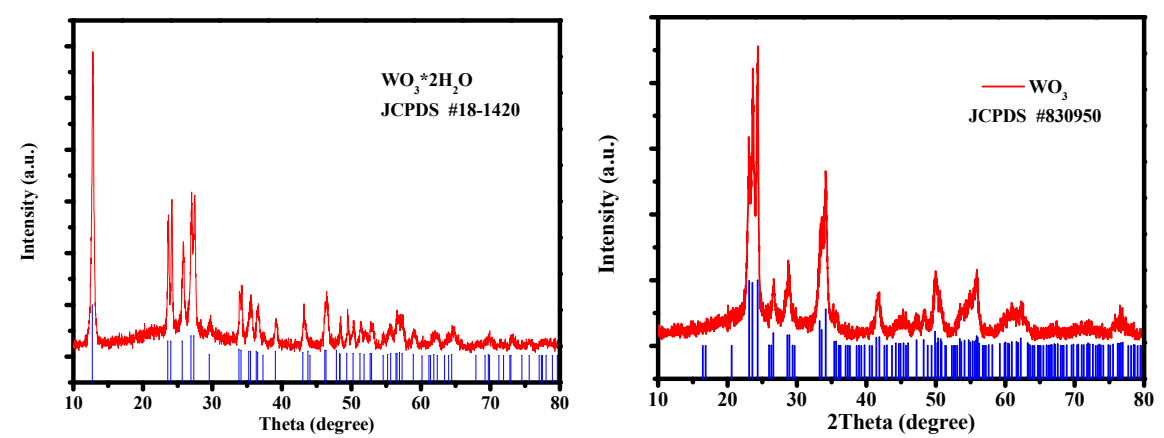

Figure S6 X-ray diffraction (XRD) patterns of the samples after three cycles 